\title{
Meta-Analysis of Economic Evaluation Studies: Data Harmonisation and Methodological Issues
}

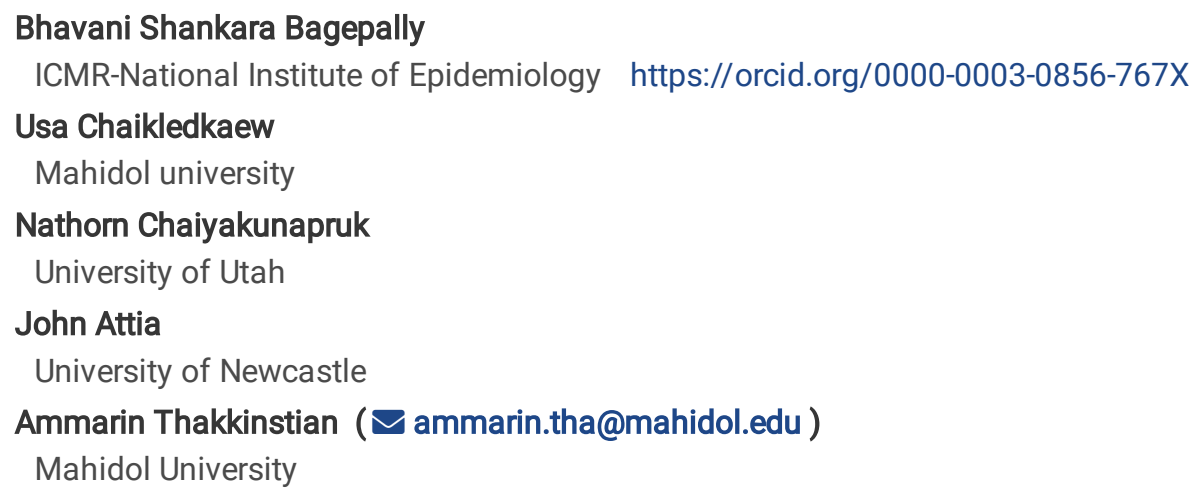




\section{Abstract}

In the context of ever-growing health expenditure and limited resources, economic evaluations aid in making evidence-informed policy decisions. Cost utility analyses (CUA) are often used in this context, but limitations include pairwise contrasts, missing contrasts, and different sources or quality of data. Synthesis of CUA data from multiple studies is therefore desirable to assist policy makers, but there are many challenging methodological issues including: inconsistent reporting of results using different economic parameters, and multiple sources of heterogeneity including: setting, time horizon, perspective, modelling approaches and assumptions, currency, willingness to pay (WTP) threshold, level of country income, and input parameters. In this paper, we provide a step by step description of the methods for data harmonisation and synthesis of aggregated data from CUA studies, as well as a framework for handling heterogeneity; we demonstrate these methods using the example of agents for type 2 diabetes. These meta-analytic methods for the synthesis of economic evidence synthesis should be useful for policy makers.

\section{Full Text}

This preprint is available for download as a PDF.

\section{Tables}

Table 1. Selected studies and its analysis scenario

\begin{tabular}{|c|c|c|c|c|c|c|c|c|c|}
\hline Study & Country & Perspective & $\begin{array}{l}\text { Time } \\
\text { horizon }\end{array}$ & $\begin{array}{l}\text { Reference } \\
\text { year }\end{array}$ & Threshold & ICER & $\begin{array}{l}\text { Measure of } \\
\text { dispersion }\end{array}$ & $\begin{array}{l}\text { CE- } \\
\text { Plane }\end{array}$ & Scenario \\
\hline Sinha [1] & USA & Payers & Life-time & 2008 & $\$ 50,000$ & yes & none & no & 5 \\
\hline Davies [2] & UK & Payers & Life-time & 2008 & $£ 20,000$ & yes & SD & no & 3 \\
\hline Guillermin [3] & USA & Payers & 35 -yrs & NA & $\$ 50,000$ & no & SD & no & 5 \\
\hline Lee [4] & USA & Payers & $35-y r s$ & 2011 & $\$ 50,000$ & yes & SD & no & 3 \\
\hline $\begin{array}{l}\text { Mezquita- } \\
\text { Raya [5] }\end{array}$ & Spain & Payers & Life-time & 2012 & $€ 30,000$ & yes & SD & yes & 3 \\
\hline $\begin{array}{l}\text { Steen- } \\
\text { Carlsson [6] }\end{array}$ & Sweden & Societal & Life-time & 2013 & $\begin{array}{l}\text { SEK } \\
500,000\end{array}$ & yes & NA & yes & 4 \\
\hline Perez [7] & Spain & Payers & Life-time & 2012 & $€ 30,000$ & yes & SD & no & 3 \\
\hline Bruhn [8] & USA & Payers & $50-y r s$ & 2014 & $\$ 50,000$ & yes & SD & yes & 3 \\
\hline Roussel [9] & France & Payers & Life-time & 2013 & $€ 30,000$ & yes & $95 \% \mathrm{Cl} \& \mathrm{SD}$ & yes & 3 \\
\hline Barnett [10] & UK & Payers & Life-time & 2016 & $£ 20,000$ & yes & SD & yes & 3 \\
\hline
\end{tabular}

Cl, confidence interval; NA, Not available; SD, Standard deviation

Table 2. Descriptive of the mean cost and QALY along with their incremental data of comparison between GLP1a vs DPP4i. 


\begin{tabular}{|c|c|c|c|c|c|c|c|c|}
\hline \multirow[t]{2}{*}{ Author } & \multicolumn{4}{|l|}{ Cost } & \multicolumn{3}{|l|}{ QALY } & \multirow[t]{2}{*}{ ICER } \\
\hline & Currency & GLP1 & DPP4i & $\Delta \mathrm{C}$ & GLP1 & DPP4i & $\Delta \mathrm{E}$ & \\
\hline Sinha [1] & US \$ & 170799 & 167163 & 3636 & 15.2998 & 15.3335 & -0.0337 & -107893 \\
\hline Davies [2] & $£$ & $21793 \pm 544$ & $19951 \pm 521$ & $1842 \pm 751$ & $7.52 \pm 0.11$ & $7.34 \pm 0.11$ & $0.19 \pm 0.15$ & 10158 \\
\hline $\begin{array}{l}\text { Guillermin } \\
\text { [3] }\end{array}$ & US \$ & 55647 & 57862 & -2215 & $9.56 \pm 0.12$ & $9.28 \pm 0.12$ & $0.284 \pm 0.172$ & -7799 \\
\hline Lee [4] & US \$ & $81444 \pm 1079$ & $76262 \pm 1061$ & 5182 & $8.825 \pm 0.117$ & $8.624 \pm 0.115$ & 0.201 & 31488 \\
\hline $\begin{array}{l}\text { Mezquita- } \\
\text { Raya[5] }\end{array}$ & $€$ & $54684 \pm 1250$ & $52387 \pm 1346$ & 2297 & $9.04 \pm 0.13$ & $8.87 \pm 0.11$ & 0.17 & 13266 \\
\hline $\begin{array}{l}\text { Steen- } \\
\text { Carlsson } \\
{[6]}\end{array}$ & SEK & 1360715 & 1304092 & 56624 & 10.53 & 10.15 & 0.38 & 154226 \\
\hline Perez [7] & $€$ & $56628 \pm 1323$ & $52450 \pm 1394$ & 4177 & $9.239 \pm 0.121$ & $8.838 \pm 0.121$ & 0.4 & 10436 \\
\hline Bruhn [8] & US \$ & $140806 \pm 1948$ & $138583 \pm 2071$ & 2223 & $9.618 \pm 0.125$ & $9.517 \pm 0.130$ & 0.101 & 22094 \\
\hline $\begin{array}{l}\text { Roussel } \\
{[9]}\end{array}$ & $€$ & $43031 \pm 1532$ & $40472 \pm 1513$ & $\begin{array}{l}2558 \\
(2427,2689) \\
\star\end{array}$ & $10.09 \pm 0.13$ & $9.84 \pm 0.13$ & $\begin{array}{l}0.25(0.24 \\
0.26)^{\star}\end{array}$ & 10275 \\
\hline $\begin{array}{l}\text { Barnett } \\
{[10]}\end{array}$ & $£$ & $24737 \pm 739$ & $22362 \pm 725$ & 2375 & $9.18 \pm 0.12$ & $9.02 \pm 0.11$ & 0.15 & 15423 \\
\hline
\end{tabular}

Values in cell are mean \pm standard deviation; $* 95 \% \mathrm{Cl}, \Delta \mathrm{C}$-incremental cost, $\Delta \mathrm{E}$-incremental QALY, GLP1a-Glucagon-like peptide 1 agonists, DPP4i- Dipeptidyl peptidase-4 inhibitors

Table 3. Describe incremental net benefit comparing GLP1i with DPP4i along with variance

\begin{tabular}{|lll|}
\hline Authors & $\begin{array}{l}\text { Mean INB } \\
\text { (PPP adjusted US \$) }\end{array}$ & Variance INB \\
\hline Sinha [1] & $-6,058$ & $7,58,90,095$ \\
\hline Davies [2] & 3,063 & $3,05,70,369$ \\
\hline Guillermin [3] & 18,452 & $7,58,90,095$ \\
\hline Lee [4] & 5,267 & $7,58,90,095$ \\
\hline Mezquita-Raya [5] & 1,529 & $3,66,23,523$ \\
\hline Steen-Carlsson [6] & $-11,643$ & $4,31,66,49,739$ \\
\hline Perez [7] & 12,007 & $7,18,90,710$ \\
\hline Bruhn [8] & 3,077 & $9,68,23,864$ \\
\hline Roussel [9] & 6,373 & $5,54,03,868$ \\
\hline Barnett [10] & 1,172 & $2,45,24,439$ \\
\hline
\end{tabular}

INB, incremental net benefit; PPP, purchasing power parity, GLP1a-Glucagon-like peptide 1 agonists, DPP4i- Dipeptidyl peptidase-4 inhibitors

Table 4: The subgroup analysis results of pooing INB between the GLP1 agonists and DPP4 inhibitors 


\begin{tabular}{|llllll|}
\hline Subgroup analysis & No. of comparisons & Pooled INB (US\$) & $\mathbf{9 5 \%}$ Cl & p-value & $\mathbf{I}^{2}$ (\%) \\
\hline Threshold & & & & & \\
\hline$<$ Median $(\$ 49,325)$ & 5 & $3,554.00$ & $-1,825.34$ to 8933.34 & 0.829 & 0.0 \\
\hline$\geq$ Median $(\$ 49,325)$ & 5 & $5,226.56$ & -3530.74 to $13,983.86$ & 0.393 & 2.4 \\
\hline Time Horizon & & & & & \\
\hline Life time & 7 & $2,663.36$ & -2463.30 to 7790.01 & 0.852 & 0.0 \\
\hline Non-lifetime & 3 & $9,386.72$ & -846.73 to 19620.17 & 0.424 & 0.0 \\
\hline Source of effectiveness & 4 & & & & \\
\hline Multiple study & 4 & $1,538.51$ & $-8,049.60$ to $11,126.62$ & 0.742 & 0.0 \\
\hline Single study & 6 & $4,745.01$ & -473.58 to $9,963.59$ & 0.534 & 0.0 \\
\hline
\end{tabular}

\section{Figures}
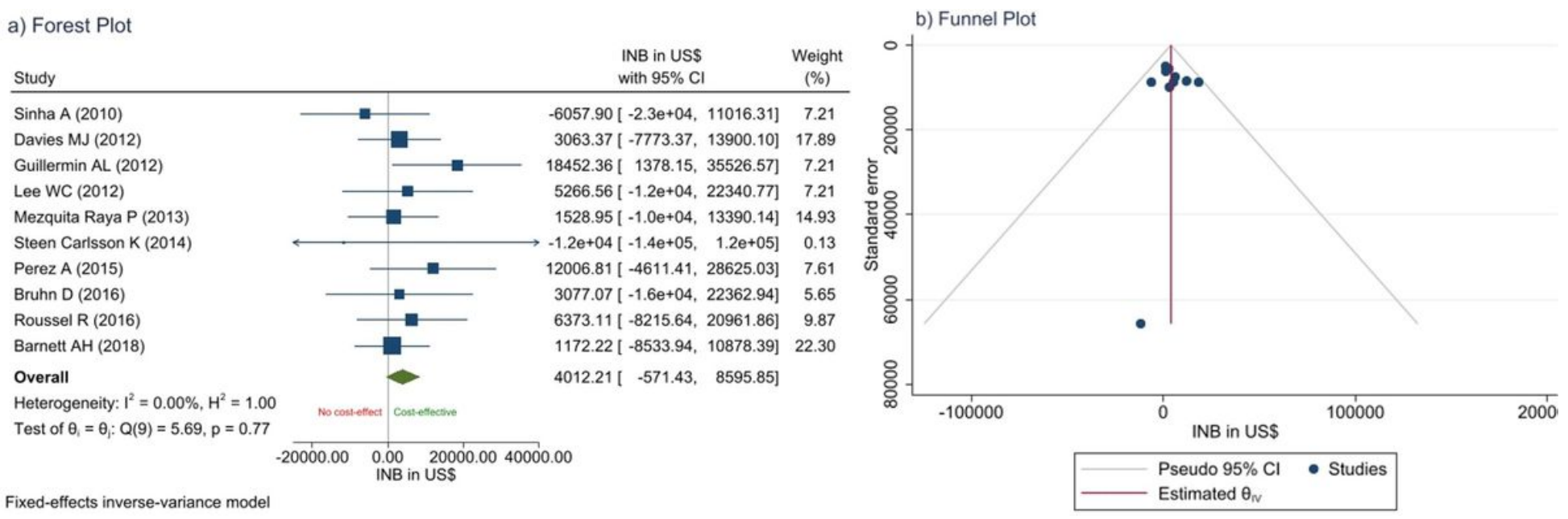

\section{Figure 1}

a) Forrest plot of pooling INBs of GLP1 vs DPP4i; b) Funnel plot of pooling INB of GLP1 vs DPP4i. 